\title{
Independent and joint effect of type 2 diabetes and gastric and hepatobiliary diseases on risk of pancreatic cancer risk: 10-year follow-up of population-based cohort
}

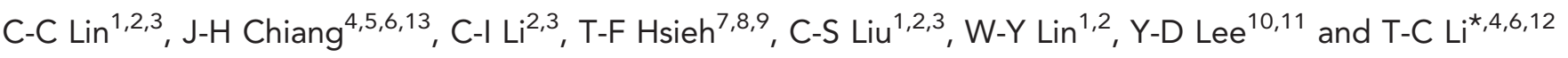

${ }^{1}$ Department of Family Medicine, China Medical University Hospital, Taichung, Taiwan; ${ }^{2}$ School of Medicine, College of Medicine, China Medical University, Taichung, Taiwan; ${ }^{3}$ Department of Medical Research, China Medical University Hospital, Taichung, Taiwan; ${ }^{4}$ Graduate Institute of Biostatistics, College of Management, China Medical University, Taichung, Taiwan; ${ }^{5}$ Management Office for Health Data, China Medical University Hospital, Taichung, Taiwan; ${ }^{6}$ Research Center for Chinese Medicine and Acupuncture, China Medical University, Taichung, Taiwan; ${ }^{7}$ Division of Urology, Department of Surgery, Buddhist Tzu Chi General Hospital, Taichung Branch, Taichung, Taiwan; ${ }^{8}$ School of Medicine, Buddhist Tzu Chi University, Hualien, Taiwan; ${ }^{9}$ Graduate Institute of Clinical Medical Science, College of Medicine, China Medical University, Taichung, Taiwan; ${ }^{10}$ Department of Psychiatry, Medical College, National Cheng-Kung University, Tainan, Taiwan; ${ }^{11}$ Global Development and Medical Affairs, BristolMyers Squibb (Taiwan) Ltd, Taipei, Taiwan and ${ }^{12}$ Department of Healthcare Administration, College of Medical and Health Science, Asia University, Taichung, Taiwan
\end{abstract}

Background: Type 2 diabetes mellitus, gastric and hepatobiliary comorbidities, and cancer share common risk factors: for example, tobacco, obesity, physical inactivity, high calorie intake, and metabolic disorders. Prior studies find type 2 diabetes and gastric and hepatobiliary comorbidities heightening risk of pancreatic cancer. Yet joint association of type 2 diabetes mellitus and gastric and hepatobiliary comorbidities on pancreatic cancer risk has not been assessed.

Methods: This study rates independent/joint effects of type 2 diabetes as well as gastric and hepatobiliary comorbidity on pancreatic cancer risk for a retrospective population-based cohort of 166850 type 2 diabetics identified in 1997-1998 and followed for 10-11 years, comparing their cancer incidence with that of 166850 non-diabetics matched for age, gender, and locale. Time-dependent Cox's proportional hazards model evaluted joint association of type 2 diabetes and chronic conditions on pancreatic cancer risk.

Results: A total of 1178 subjects were newly diagnosed with pancreatic cancer during follow-up, with incidence rates of 0.49 per 1000 person-years in type 2 diabetes and 0.26 per 1000 person-years in the non-diabetics. We observed greater magnitude of hazard ratios (HRs) of pancreatic cancer for patients with type 2 diabetes along with acute alcoholic hepatitis, acute pancreatitis, cholecystitis, and gastric ulcer compared with patients without type 2 diabetes or counterpart comorbidity (HR: 1.36, 95\% confidence interval (CI): 1.19-1.56; 1.74, 1.23-2.45; 9.18, 7.44-11.33; and 2.31, 1.98-2.70, respectively). Main effects of type 2 diabetes were all statistically with narrow $95 \% \mathrm{Cl}$ and remained similar across risk stratification with various comorbidities: range 1.59-1.80.

Conclusions: Our study demonstrates that pre-existing type 2 diabetes, acute alcoholic hepatitis, acute pancreatitis, cholecystitis, and gastric ulcer independently or jointly predict subsequent pancreatic cancer risk. Clinicians must recognise burden of these gastric and hepatobiliary comorbidities and keep clinically vigilant for their diagnosis.

*Correspondence: Professor T-C Li; E-mail: tcli@mail.cmu.edu.tw

${ }^{13}$ Equal contribution as the first author.

Received 13 May 2014; revised 29 August 2014; accepted 7 September 2014; published online 2 October 2014

(c) 2014 Cancer Research UK. All rights reserved 0007 - 0920/14 
Cancer has ranked number-one cause of death in Taiwan since 1982, and average age of those diagnosed with it shows trend a downward. Taiwan's Department of Health national statistics report cited pancreatic cancer in 2012 ranking ninth highest overall for cancer death among the Taiwanese population: 6.4 per 100000 (Ministry of Health and Welfare, 2012).

Type 2 diabetes mellitus, gastric and hepatobiliary comorbidity, and cancers share common risk factors, including tobacco, alcohol, obesity, physical inactivity, a high calorie (particularly saturated fat) intake, and metabolic disorders (Giovannucci and Michaud, 2007; Xue and Michels, 2007; Cornier et al, 2008). The possible biological mechanism that type 2 diabetes and gastric and hepatobiliary comorbidity contributed to elevated risk of developing pancreatic cancer may be via inflammatory (Lee et al, 2008). Prior bench studies show that dysregulation of tuberous sclerosis 1 (TSC1)/TSC2/mTOR signalling pathway by $\mathrm{I} \kappa \mathrm{B}$ kinase $\beta(\mathrm{IKK} \beta)$ is a common molecular switch for both cancer pathogenesis and diet- and obesity-induced insulin resistance (Lee et al, 2008). Obesity-derived chronic inflammation with insulin resistance is also associated with gastric and hepatobiliary comorbidity (Farrow and Evers, 2002).

Previous population studies using rate standardisation indicate pancreatic cancer strongly correlating with type 2 diabetes (Hemminki et al, 2010; Atchison et al, 2011; Ben et al, 2011a, b; Lin et al, 2014). Several studies further rated association of pancreatic cancer with type 2 diabetes (Coughlin et al, 2004; Jee et al, 2005; Ogunleye et al, 2009; Stevens et al, 2009; Chodick et al, 2010; Chen et al, 2011) to elucidate such association after adjusting for traditional risk factors in across ethnicities (Larsson et al, 2005; Ogunleye et al, 2009; Stevens et al, 2009; Chodick et al, 2010; Lam et al, 2011), but this line of study conducted in a Chinese population was limited (Chen et al, 2011). Since 2000, several case-control and cohort studies explored the association between type 2 diabetes and pancreatic cancer (Frye et al, 2000; Gapstur et al, 2000; Lund Nilsen et al, 2000; Silverman, 2001; Lin et al, 2002; Stolzenberg-Solomon et al, 2002; Bonelli et al, 2003; Inoue et al, 2003; Rulyak et al, 2003; Batty et al, 2004; Coughlin et al, 2004; Jee et al, 2005). One casecontrol study reported that type 2 diabetes was associated with 2.8fold increased risk of pancreatic cancer (Bonelli et al, 2003) whereas two case-control studies did not observe a significant association (Frye et al, 2000; Silverman, 2001). Similarly, findings of most prior cohort studies supported this association with a relative risk ranging from 1.48 to 3.99 (Gapstur et al, 2000; Lin et al, 2002; StolzenbergSolomon et al, 2002; Inoue et al, 2003; Rulyak et al, 2003; Batty et al, 2004; Coughlin et al, 2004; Jee et al, 2005). Only two cohort studies did not support the hypothesis that patients with type 2 diabetes are more likely to develop pancreatic cancer (Lund Nilsen et al, 2000). However, none of these studies considered gastric and hepatobiliary comorbidity.

Some researchers exploring links between type 2 diabetes and pancreatic cancer took gastric and hepatobiliary comorbidity such as acute pancreatitis, chronic pancreatitis, alcoholic liver disease, pancreatic pseudocyst, cholelithiasis (Bansal and Sonnenberg, 1995), gallbladder disease (Bracci et al, 2009), hepatitis B, hepatitis C, cholecystitis, cholangitits, cholelithiasis, gastric ulcer, and duodenal ulcer (Chen et al, 2011) into account; none considered joint effect with type 2 diabetes on pancreatic cancer. This population-based study examined independent and joint association of type 2 diabetes and gastric or hepatobiliary comorbidity on pancreatic cancer risk in Taiwan.

\section{MATERIALS AND METHODS}

A single-payer National Health Insurance (NHI) program was implemented in March 1995. As of 2007, 22.60 million of Taiwan's 22.96 million populations enrolled. Nation-run Bureau of National
Health Insurance (BNHI) contracted with $97 \%$ of hospitals as well as $92 \%$ of clinics nationwide (Ministry of Health and Welfare, 2012). Bureau of National Health Insurance performs quarterly expert reviews on random samples of every 50-100 ambulatory and in-patient claims in each hospital and clinic. False diagnosis reports entail a high penalty.

Our population-based cohort study used type 2 diabetes patients (aged $\geqslant 20$ years) identified in 1997-1998 and followed up through 31 December 2010 or until the first manifestation of pancreatic cancer. Population with type 2 diabetes should have at least three ambulatory claims or at least one in-patient claim with diagnosis of ICD-9-CM code 250 or A-code A181 in 1997-1998. To exclude those individuals with type 1 diabetes, we have done two steps. First, we identify all individuals with type 1 diabetes from Registry for Catastrophic Illness database. Second, we excluded those individuals with type 1 diabetes identified in the first step from our study cohort with diabetes. We excluded subjects with type 1 diabetes $(N=3750)$ and any cancer type at baseline $(N=135060)$ from 633680 patients with type 2 diabetes, aged $<20$ years $(N=17679)$, and/or with incomplete information on gender as well as residential area $(N=4212)$. This left 472979 patients with type 2 diabetes. Subjects from the general population were selected from Longitudinal Health Insurance Database 2005 (LHID2005) created by National Health Research Institutes (NHRI) by randomly sampling 1000000 beneficiaries from Registry for Beneficiaries data files for year 2005. Our sample proved representative of the overall population (Tseng et al, 2012). Database contained all longitudinal reimbursement information of this random sample from 1996 to the end of 2010 . There were a total of 874053 subjects in 1997-1998 in the file of LHID2005; excluding those with type 1 diabetes $(N=363)$ from Registry for Catastrophic Illness database, cancer $(N=28550)$, diabetes $(N=121227)$, and/or age under 20 years $(N=304293)$ in 1997 1998 , as well as those with incomplete information on sex and residential area $(N=11)$, left 419609 eligible non-diabetics. The same eligibility criteria applied to each group, yet distribution of age, gender, and residential areas was unbalanced between groups. To bolster comparability between cases of type 2 diabetes and persons without diabetes, we randomly selected equal numbers of type 2 diabetics and non-diabetics from each stratum of combination of age (5-year groups), sex and residential areas (25 counties or cities). This left 166850 patients with type 2 diabetes and individuals without any diabetes in 1997-1998 (Figure 1). Baseline or index date for type 2 diabetes group was date of first outpatient visit or in-patient admission. For non-diabetics, index date was randomly assigned from 1 January 1997 to 31 December 1998 according to index date distribution of type 2 diabetes group.

All data sets can be interlinked via individual personal identification number (PIN), which was scrambled cryptographically by NHIRD to protect enrollee's privacy. Ambulatory care claims contain the individual's gender and birthday, date of visit, and codes for International Classification of Diseases, Ninth Revision, Clinical Modification (ICD-9-CM) codes, or A-codes for three primary diagnoses. In-patient claims contain ICD-9-CM codes for principal diagnosis up to four secondary diagnoses. The primary outcome was a new diagnosis of pancreatic cancer (ICD9-CM code 157; A-code A096), including all malignant pancreatic tumours such as adenocarcinoma and pancreatic endocrine tumours after index date during the follow-up. Gastric and hepatobiliary comorbidity comprised acute or chronic pancreatitis, acute hepatitis, alcoholic fatty liver or cirrhosis, acute alcoholic hepatitis, cholelithiasis, morbid obesity, alcohol dependence syndrome, pseudocyst of pancreas, jaundice, hepatitis B, hepatitis $\mathrm{C}$, cholecystitis, cholangitis, gastric ulcer, and duodenal ulcer. Each individual patient's status of gastric and hepatobiliary comorbidities at each year was identified for time-dependent data analysis. Sociodemographic factors include age, gender, insurance premium, 


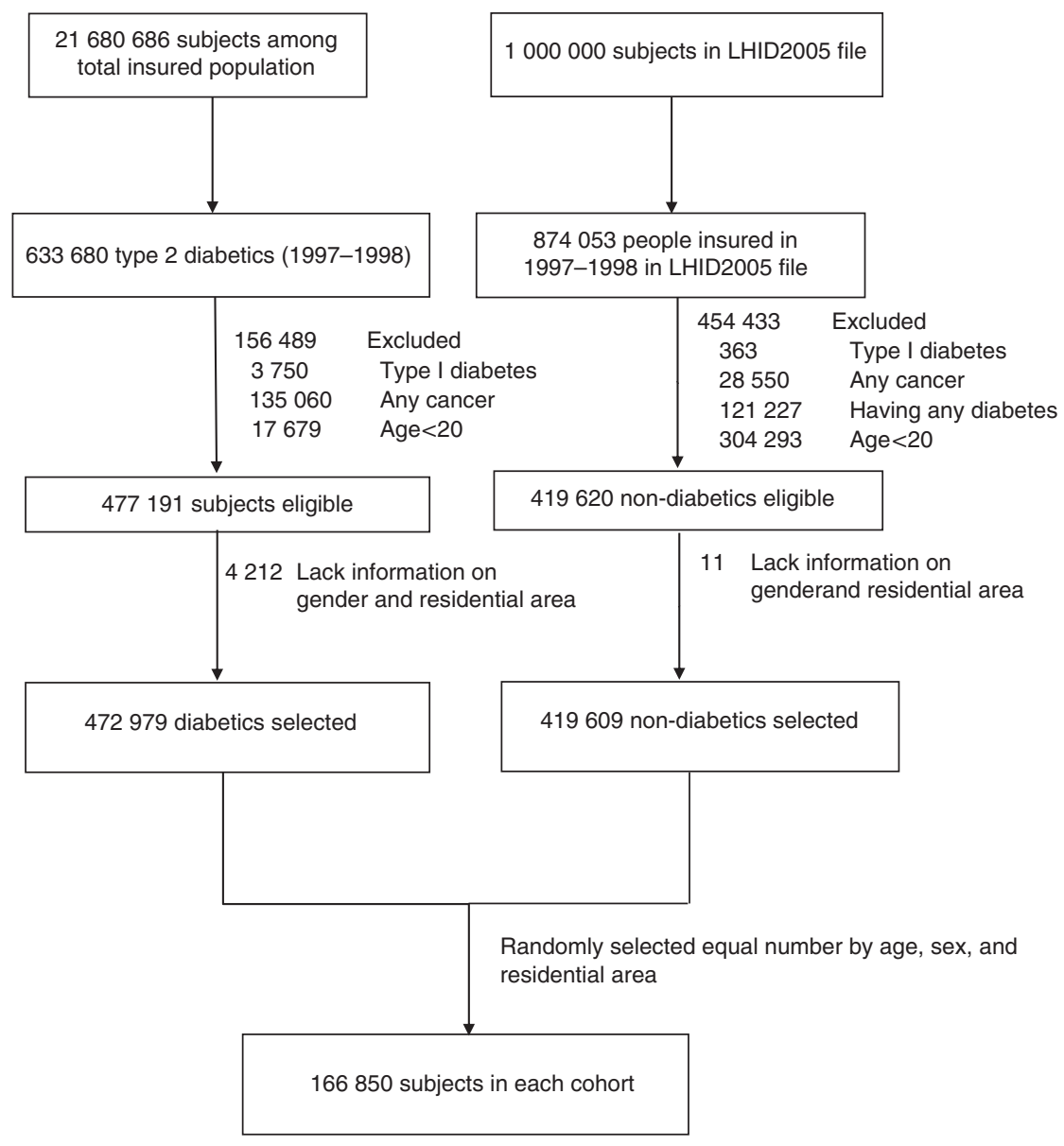

Figure 1. Flowchart of recruitment procedures.

and urbanisation degree of residential area. Age was divided into 17 groups with 5 -year intervals from 20 to $>90$ years. Gender was categorised as male and female, insurance according to median of amounts of premiums, in which median value for these two groups was NT\$19200 from 1997 to 1998. We used an urbanisation indicator developed by Liu et al (2006), who categorised 365 Taiwan towns as per degrees of urbanisation: high- and mediumdensity urban areas, newly developed area, general area, ageingsociety area, rural area, and non-developed area.

We compared baseline characteristic between subjects with and without type 2 diabetes using standardised mean differences. All standardised mean differences were less than 0.1 s.d., indicating a negligible difference in means or proportions between two groups. To explore the joint effect of type 2 diabetes and each gastric and hepatobiliary comorbidity, three dummy variables were created. Using individuals without type 2 diabetes and comorbidity as reference group, these three dummy variables measured the effects of type 2 diabetes only, comorbidity only, and combined type 2 diabetes and comorbidity. Time-dependent Cox's proportional hazards model evaluted hazards ratio (HR) of type 2 diabetes on pancreatic cancer. Time-dependent covariates included acute or chronic pancreatitis, acute hepatitis, alcoholic fatty liver or cirrhosis, acute alcoholic hepatitis, cholelithiasis, morbid obesity, alcohol dependence syndrome, pseudocyst of pancreas, jaundice, hepatitis B, hepatitis $\mathrm{C}$, cholecystitis, cholangitis, gastric ulcer, and duodenal ulcer. Outcome variable was timed to occurrence of newly diagnosed pancreatic cancer during study period. A subject was censored if he or she died, developed non-pancreatic cancer, or else did not develop pancreatic cancer before end of the study. For selected independent variables that result in a 'best' model, we followed four steps (Hosmer and Lemeshow, 2000). First, selection process began with a careful univariable analysis of each variable. Second, on completion of univariate analysis, we selected variable whose univariable test has a $P$-value of $<0.25$ (Bendel and Afifi, 1977; Mickey and Greenland, 1989) as a candidate for our multivariable model. Third, enter candidate variables into the multivariable model. With some comorbidity variables highly collinear, we estimated their regression coefficients and compared these for significance. Only one such highly correlated variable remained in a multivariate Cox model. Finally, after refining a main effects model, we checked assumption of Cox's proportional hazard model for all variables in our multivariate model, further examining interactions between independent variables.

\section{RESULTS}

Table 1 shows baseline characteristics according to status of type 2 diabetes. Subjects with and without type 2 diabetes had similar distributions of gender, age, and residential area. Percentages of female and male were $44.44 \%$ and $55.56 \%$. Compared with nondiabetics, type 2 diabetics had higher proportion of ageing society area, insurance premium less than NT $\$ 19,200$, gastric and hepatobiliary comorbidities for acute alcoholic hepatitis, acute hepatitis, acute pancreatitis, alcoholic fatty liver and/or cirrhosis, nonalcoholic fatty liver, cholelithiasis, chronic pancreatitis, morbid obesity, alcohol dependence, pseudocyst of pancreas, jaundice, hepatitis $B$, hepatitis $C$, and cholangitis.

A total of 1178 subjects were newly diagnosed with pancreatic cancer during follow-up, with incidence rates of 0.49 per 1000 person-years in type 2 diabetics and 0.26 per 1000 person-years in 
Table 1. Baseline characteristics of study cohorts by sociodemographic status and comorbidity in Taiwan

\begin{tabular}{|c|c|c|c|}
\hline & Non-diabetes (\%) $(N=166 \quad 850)$ & Type 2 diabetes $(\%)(N=166 \quad 850)$ & Standardised mean difference \\
\hline \multicolumn{4}{|l|}{ Sex } \\
\hline Female & $74148(44.44 \%)$ & $74148(44.44 \%)$ & 0.00 \\
\hline Male & $92702(55.56 \%)$ & $92702(55.56 \%)$ & 0.00 \\
\hline Age, mean (s.d.) & $50.47(12.07)$ & $50.71(11.80)$ & -0.02 \\
\hline \multicolumn{4}{|l|}{ Urbanisation degree } \\
\hline High density urban area & 46967 (28.48\%) & $47128(28.61 \%)$ & 0.00 \\
\hline Medium density urban area & $47521(28.81 \%)$ & $48861(29.66 \%)$ & -0.02 \\
\hline Newly developed area & $28844(17.49 \%)$ & $28045(17.02 \%)$ & 0.01 \\
\hline General area & $24458(14.83 \%)$ & $24262(14.73 \%)$ & 0.00 \\
\hline Ageing society area & $3440(2.09 \%)$ & 3655 (2.22\%) & -0.01 \\
\hline Rural area & 7264 (4.40\%) & $6773(4.11 \%)$ & 0.01 \\
\hline Non-developed area & $6437(3.90 \%)$ & $6028(3.66 \%)$ & 0.01 \\
\hline \multicolumn{4}{|l|}{ Insurance premium } \\
\hline INS-AMT $<19200$ & $41195(24.69 \%)$ & $44469(26.65 \%)$ & -0.04 \\
\hline $19200 \leqq I N S-A M T$ & $125655(75.31 \%)$ & $122381(73.35 \%)$ & 0.04 \\
\hline \multicolumn{4}{|l|}{ Comorbidity } \\
\hline Acute alcoholic hepatitis & $19855(11.90 \%)$ & $37973(22.76 \%)$ & -0.29 \\
\hline Acute hepatitis & $177(0.11 \%)$ & $91(0.05 \%)$ & 0.02 \\
\hline Acute pancreatitis & $186(0.11 \%)$ & $252(0.15 \%)$ & -0.01 \\
\hline Alcoholic fatty liver & 19864 (11.91\%) & 37997 (22.77\%) & -0.29 \\
\hline Non-alcoholic fatty liver disease & $571(0.34 \%)$ & $391(0.23 \%)$ & 0.02 \\
\hline Alcoholic cirrhosis of liver & $19837(11.89 \%)$ & $37990(22.77 \%)$ & -0.29 \\
\hline Cholelithiasis & $1126(0.67 \%)$ & $407(0.24 \%)$ & 0.06 \\
\hline Chronic pancreatitis & 37 (0.02\%) & $245(0.15 \%)$ & -0.04 \\
\hline Morbid obesity & $113(0.07 \%)$ & $605(0.36 \%)$ & -0.06 \\
\hline Alcohol dependence syndrome & 317 (0.19\%) & 314 (0.19\%) & 0.00 \\
\hline Pseudocyst of pancreas & 6 (0.00\%) & $13(0.01 \%)$ & -0.01 \\
\hline Jaundice & $62(0.04 \%)$ & $25(0.01 \%)$ & 0.02 \\
\hline Hepatitis B & $1981(1.19 \%)$ & $2248(1.35 \%)$ & -0.01 \\
\hline Hepatitis C & $1774(1.06 \%)$ & $2141(1.28 \%)$ & -0.02 \\
\hline Cholecystitis & $3740(2.24 \%)$ & $1953(1.17 \%)$ & 0.08 \\
\hline Cholangitis & $143(0.09 \%)$ & $43(0.03 \%)$ & 0.03 \\
\hline Gastric ulcer & 41906 (25.12\%) & $27391(16.42 \%)$ & 0.21 \\
\hline Duodenal ulcer & 41976 (25.16\%) & 27408 (16.43\%) & 0.22 \\
\hline
\end{tabular}

the non-diabetics. Table 2 displays uni- and multivariate timedependent Cox's proportional hazard models in a cohort of type 2 diabetics vs general population during 1999-2010. Our model building strategy determined a final multivariable model shown in Table 2. Significant adjusted HRs of pancreatic cancer in Cox proportional hazard models were type 2 diabetes (HR: 1.50, 95\% CI: $1.32-1.71)$, age (per 5 years) $(1.18,1.75-1.21)$, men $(1.31,1.16-$ $1.47)$, acute alcoholic hepatitis $(1.36,1.19-1.56)$, acute pancreatitis $(1.74,1.23-2.45)$, cholelithiasis $(0.46,0.33-0.62)$, chronic pancreatitis $(2.55,1.69-3.87)$, cholangitis $(9.18,7.44-11.33)$, gastric ulcer (2.31, 1.98-2.70), and duodenal ulcer (0.51, 0.43-0.60).

In sensitivity analysis by calculating follow-up starting 1 or 3 years after baseline to rule out the possibility of reverse causaility, type 2 diabetes also showed strong linkage with pancreatic cancer during follow-up starting 1 year after baseline (HR: 1.57, 95\% CI: $1.38-1.78, P<0.0001$ ), as well as follow-up beginning 3 years later (HR: 1.43 , 95\% CI: 1.25-1.63). Figure 2 shows adjusted HR of pancreatic cancer for joint effects of type 2 diabetes and acute alcoholic hepatitis, acute pancreatitis, nonalcoholic fatty liver disease, cholelithiasis, cholecystitis, cholangitis, gastric ulcer, and duodenal ulcer. We observed greater magnitude of HRs of pancreatic cancer for type 2 diabetics with acute alcoholic hepatitis, acute pancreatitis, cholecystitis, cholangitis and gastric ulcer $v s$ patients with neither type 2 diabetes nor counterpart comorbidity $(2.15,1.81-2.56 ; 6.55,2.52-17.04 ; 3.34,2.16-5.16 ; 7.30,1.01-52.78$ and $4.41,2.38-8.20$, respectively). Independent effects of type 2 diabetes were all statistically significant, with narrow 95\% CI, and remained similar across risk stratification with comorbidity (range 1.59-1.80). Factors exerting significant independent effect were acute alcoholic hepatitis $(1.35,1.18-1.55)$, cholecystitis $(2.02,1.47-$ $2.77)$, and gastric ulcer $(2.85,1.56-5.18)$. Due to limited number of study subjects with chronic pancreatic or alcohol dependence syndrome, joint association of type 2 diabetes with these was not evaluated. In addition, we detected significant interaction of type 2 diabetes with cholelithiasis, gastric ulcer, and duodenal ulcer ( $P=0.027,0.007$, and 0.001 , respectively).

\section{DISCUSSION}

This study evaluated association between type 2 diabetes and pancreatic cancer among a large prospective cohort to find positive association between type 2 diabetes and pancreatic cancer during 10-11 year follow-up. Our study's findings show that patients with type 2 diabetes are associated with increased risk of pancreatic cancer; sensitivity analyses remained similar after adjustment for sociodemography and time-dependent comorbidity, to rule out reverse causality. We observed significant joint associations between type 2 diabetes and acute alcoholic hepatitis, acute pancreatitis, cholecystitis, and gastric ulcer on pancreatic cancer risk.

Most previous studies correlating diabetes with pancreatic cancer indicate type 2 diabetes as a risk factor (Coughlin et al, 2004; Jee et al, 2005; Larsson et al, 2005; Inoue et al, 2006; Khan et al, 2006; Ogunleye et al, 2009; Stevens et al, 2009; Chodick et al, 2010; Chen et al, 2011; Lam et al, 2011), although one conducted in Japan observed no such association (Khan et al, 2006). Temporal association between diabetes and pancreatic cancer has been questioned; some studies had too short a follow-up (Chen et al, 2011; Lam et al, 2011). It is likely that pancreatic cancer could lead to diabetes by abnormal glucose metabolism (so-called 'reversecausality' or effect-cause). The American Society of Clinical 
Table 2. Risk factors of pancreatic cancer from uni- and multivariate time-dependent Cox's proportional hazard models in cohort of patients with and without type 2 diabetes during 1999-2010 ( $n=1156$ for pancreatic cancer)

\begin{tabular}{|c|c|c|c|c|}
\hline Variable & Crude HR H $^{\mathrm{a}}(95 \% \mathrm{Cl})$ & $P$-value & Adjusted $\mathrm{HR}^{\mathrm{b}}(95 \% \mathrm{Cl})$ & $P$-value \\
\hline Type 2 diabetes & $1.61(1.43-1.83)$ & $<0.0001$ & $1.50(1.32-1.71)$ & $<0.0001$ \\
\hline Age (per 5 years) & $1.19(1.17-1.22)$ & $<0.0001$ & $1.18(1.75-1.21)$ & $<0.0001$ \\
\hline Sex (men vs women) & $1.29(1.14-1.45)$ & $<0.0001$ & $1.31(1.16-1.47)$ & $<0.0001$ \\
\hline Acute alcoholic hepatitis & $1.49(1.31-1.69)$ & $<0.0001$ & $1.36(1.19-1.56)$ & $<0.0001$ \\
\hline Acute hepatitis & $3.38(2.37-4.83)$ & $<0.0001$ & & \\
\hline Acute pancreatitis & $3.10(2.29-4.19)$ & $<0.0001$ & $1.74(1.23-2.45)$ & 0.0018 \\
\hline Alcoholic fatty liver & $1.46(1.28-1.66)$ & $<0.0001$ & & \\
\hline Non-alcoholic fatty liver disease & $1.31(1.02-1.67)$ & 0.0332 & $1.02(0.79-1.32)$ & 0.8674 \\
\hline Alcoholic cirrhosis of liver & $1.38(1.22-1.58)$ & $<0.0001$ & & \\
\hline Cholelithiasis & $1.07(0.81-1.41)$ & 0.6533 & $0.46(0.33-0.62)$ & $<0.0001$ \\
\hline Chronic pancreatitis & $4.64(3.23-6.66)$ & $<0.0001$ & $2.55(1.69-3.87)$ & $<0.0001$ \\
\hline Alcohol dependence syndrome & $1.16(0.64-2.09)$ & 0.631 & $0.81(0.44-1.50)$ & 0.4988 \\
\hline Pseudocyst of pancreas & $5.03(1.89-13.40)$ & 0.0012 & & \\
\hline Hepatitis B & $0.71(0.52-0.98)$ & 0.0392 & & \\
\hline Hepatitis C & $0.80(0.56-1.15)$ & 0.2218 & & \\
\hline Cholecystitis & $1.84(1.40-2.40)$ & $<0.0001$ & $1.21(0.90-1.62)$ & 0.204 \\
\hline Cholangitis & $11.81(9.77-14.26)$ & $<0.0001$ & 9.18 (7.44-11.33) & $<0.0001$ \\
\hline Gastric ulcer & $1.74(1.55-1.95)$ & $<0.0001$ & $2.31(1.98-2.70)$ & $<0.0001$ \\
\hline Duodenal ulcer & $1.07(0.94-1.21)$ & 0.3187 & $0.51(0.43-0.60)$ & $<0.0001$ \\
\hline
\end{tabular}

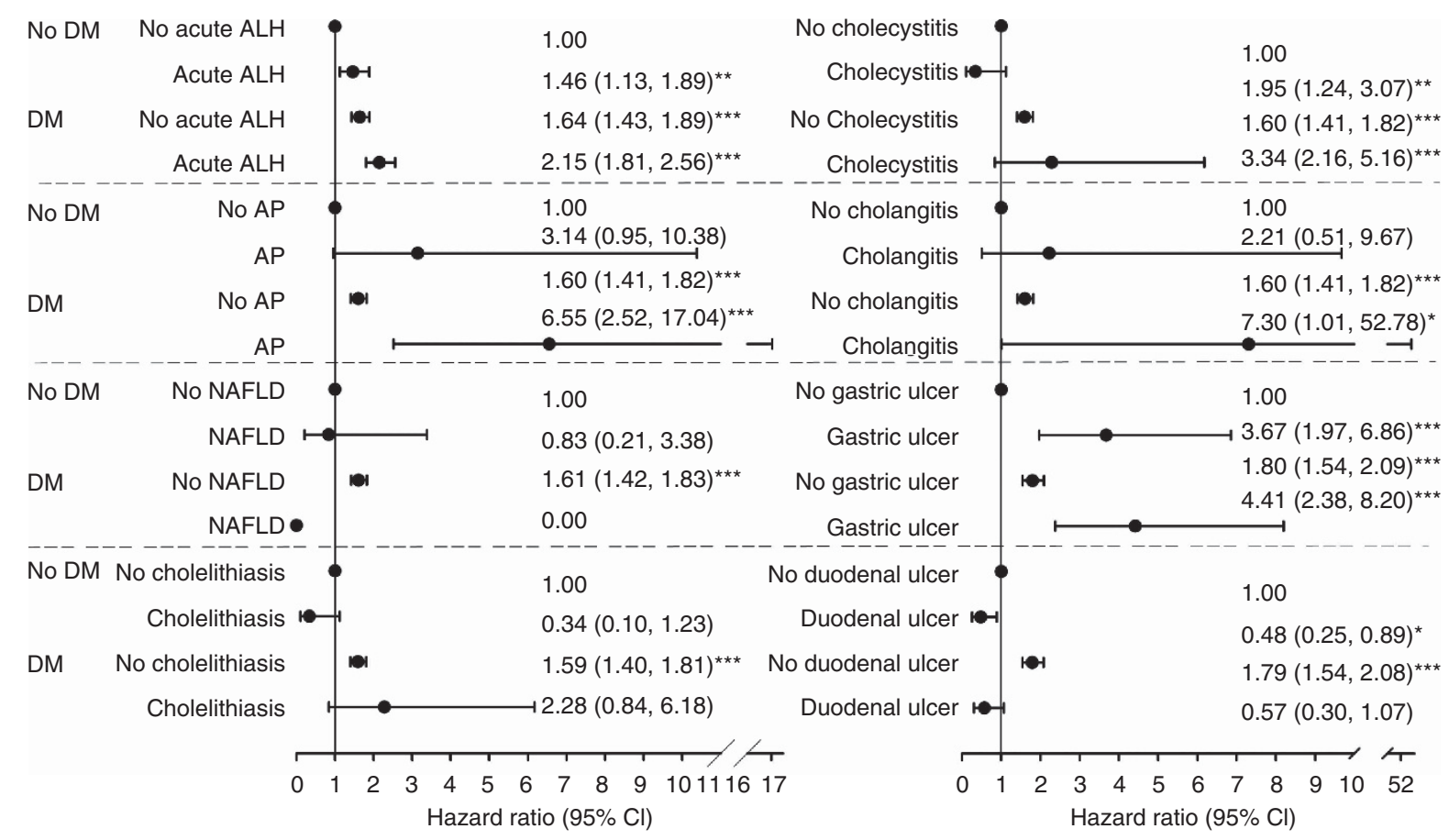

Figure 2. Joint relationship of gastric and hepatobiliary comorbidity and diabetes status on risk of pancreatic cancer. ${ }^{*} P<0.05$; ${ }^{* *} P<0.01$; ${ }^{* \star *} P<0.001$. Abbreviations: $\mathrm{DM}=$ diabetes mellitus; $\mathrm{ALH}=$ alcoholic hepatitis; $\mathrm{AP}=$ acute hepatitis; $\mathrm{NAFLD}=$ non-alcoholic fatty liver disease.

Oncology (ASCO) Annual Meeting in 2010 reported temporal association between type 2 diabetes and pancreatic cancer as unclear (Matsubayashi et al, 2011). It may be that a small portion of pancreatic cancer belonging to neuroendocrine tumour secretes diverse hormones that cause florid stomach ulcers or uncontrolled high blood pressure and diabetes (Ghaneh et al, 2007). To rule out reverse causation or effect-cause association, we evaluated this association at the outset of follow-up; 1 and 3 years after baseline, HR remained significant. This finding enhances causative association.

Type 2 diabetes has been confirmed as a risk factor for development of pancreatic cancer (Larsson et al, 2005; Inoue et al, 2006; Ogunleye et al, 2009; Stevens et al, 2009; Chodick et al, 2010; Chen et al, 2011; Lam et al, 2011), but this association has so far 
been described for its independent effect. Some previous studies accounted for acute pancreatitis, chronic pancreatitis, alcoholic liver disease, pancreatic pseudocyst and cholelithiasis (Bansal and Sonnenberg, 1995); one of them considered gallbladder disease (Bracci et al, 2009), whereas another adjusted for hepatitis B, hepatitis $\mathrm{C}$, cholecystitis, cholangitits, cholelithiasis, gastric ulcer, and duodenal ulcer (Chen et al, 2011), however, none of them consider their joint effect with type 2 diabetes on pancreatic cancer. It is unclear whether type 2 diabetes and other gastric and hepatobiliary comorbidities have significant joint roles. Our research showed history of acute alcoholic hepatitis, acute pancreatitis, cholecystitis, and gastric jointly associated with type 2 diabetes for higher risk. Data further suggest acute alcoholic hepatitis, cholecystitis, and gastric ulcer as risk factors.

There is potential misclassification error due to some undiagnosed type 2 diabetes cases in subjects without type 2 diabetes. However, this proportion would be small because Department of Health in Taiwan provides integrated on-the-site screenings for hypertension, diabetes, and hyperlipidaemia in community and primary care settings for adults aged 40 years and over and its fee was covered by NHI program. This kind of misclassification error would result in underestimation of the effect if the association between type 2 diabetes and pancreatic cancer exists, indicating that the true effect would be stronger, a lesser threat to the validity of our finding. In addition, due to limited number of diagnoses can be coded in ambulatory or in-patient claim data, patients with number of diseases more than the number of diagnoses that can be coded in the administrative claim data would be less likely to be included in our study. Thus, our study sample may not be representative of the entire population with type 2 diabetes in term of number of comorbidity. If the incidence rates of pancreatic cancer were similar across subgroups of type 2 diabetic patients with number of comorbidity, then the estimate of HR would be valid. If not, then our study finding is only applicable to type 2 diabetic patients with comorbidity similar to our study sample.

Prior studies report antidiabetic medication altering association between diabetes and pancreatic cancer (Yang, 2009). Metformin and thiazolidinediones (TZDs) protect against tumorigenesis; sulphonylurea raised risk (Yang, 2009; Giovannucci et al, 2010; Lee et al, 2011). One study demonstrated great reduction in pancreatic cancer risk for metformin users (Lee et al, 2011), sulphonylureas elevating risk (Bodmer et al, 2012). We tallied about $10 \%$ each for sulphonylurea monotherapy and metformin monotherapy (data not shown). Impact of antidiabetic medication on linkage between type 2 diabetes and pancreatic cancer can thus be ignored. Among the merits of our study: it is based on a large and representative population-based sample, hence adequate to detect independent and joint effect of type 2 diabetes and such comorbidity. Also, high coverage rate of NHI program throughout our study could minimise the number of cohort subjects lost to follow-up. Availability of NHIRD data set during follow-up can facilitate time-dependent status of comorbidities. Still, there were limitations. First, we could not obtain data of behavioural factors: for example, tobacco, alcohol, obesity, BMI, and physical activity. We did not consider them, yet it is unlikely that the effect of type 2 diabetes and gastric or hepatobiliary comorbidities would arise solely to effect of behavioural factors, which was weakly or moderately associated with pancreatic cancer. Second, it lacks histological features or pathological stages of pancreatic cancer and thus could not evaluate histological patterns, molecular markers or clinical stages. Third, it is possible that the effect of type 2 diabetes is due to reverse causality. To rule out this possibility, we performed a sensitivity analysis by calculating follow-up starting 1 or 3 years after baseline and the HRs remained significant. However, it is difficult to rule out the possibility of reverse causality due to the weak to moderate association between type 2 diabetes and pancreatic cancer.
Our study highlights pre-existing type 2 diabetes, acute alcoholic hepatitis, acute pancreatitis, chronic pancreatitis, alcohol dependence, cholecystitis, and/or gastric ulcer portending pancreatic cancer. Significant joint effects of acute alcoholic hepatitis, acute pancreatitis, cholecystitis, and gastric ulcer along with type 2 diabetes on pancreatic cancer risk were likewise noted. Clinicians must recognise burden of these gastric and hepatobiliary comorbidities and keep clinically vigilant for diagnosis.

\section{ACKNOWLEDGEMENTS}

This study is supported in part by Taiwan Ministry of Health and Welfare Clinical Trial and Research Center of Excellence (MOHW103-TDU-B-212-113002), Health and welfare surcharge of tobacco products, China Medical University Hospital Cancer Research Center of Excellence (MOHW103-TD-B-111-03, Taiwan) and China Medical University under the Aim for Top University Plan of the Ministry of Education, Taiwan.

\section{CONFLICT OF INTEREST}

The authors declare no conflict of interest.

\section{REFERENCES}

Atchison EA, Gridley G, Carreon JD, Leitzmann MF, McGlynn KA (2011) Risk of cancer in a large cohort of U.S. veterans with diabetes. Int J Cancer 128(3): 635-643.

Bansal P, Sonnenberg A (1995) Pancreatitis is a risk factor for pancreatic cancer. Gastroenterology 109(1): 247-251.

Batty GD, Shipley MJ, Marmot M, Smith GD (2004) Diabetes status and postload plasma glucose concentration in relation to site-specific cancer mortality: findings from the original Whitehall study. Cancer Causes Control 15: 873-881.

Ben Q, Cai Q, Li Z, Yuan Y, Ning X, Deng S, Wang K (2011a) The relationship between new-onset diabetes mellitus and pancreatic cancer risk: a case-control study. Eur J Cancer 47(2): 248-254.

Ben Q, Xu M, Ning X, Liu J, Hong S, Huang W, Zhang H, Li Z (2011b) Diabetes mellitus and risk of pancreatic cancer: a meta-analysis of cohort studies. Eur J Cancer 47(13): 1928-1937.

Bendel RB, Afifi AA (1977) Comparison of stopping rules in forward regression. J Am Stat Assoc 72: 46-53.

Bodmer M, Becker C, Meier C, Jick SS, Meier CR (2012) Use of antidiabetic agents and the risk of pancreatic cancer: a case-control analysis. Am J Gastroenterol 107(4): 620-626.

Bonelli L, Aste H, Bovo P, Cavallini G, Felder M, Gusmaroli R, Morandini E, Ravelli P, Briglia R, Lombardo L, De Micheli A, Pugliese V (2003) Exocrine pancreatic cancer, cigarette smoking, and diabetes mellitus: a case-control study in northern Italy. Pancreas 27: 143-149.

Bracci PM, Wang F, Hassan MM, Gupta S, Li D, Holly EA (2009) Pancreatitis and pancreatic cancer in two large pooled case-control studies. Cancer Causes Control 20(9): 1723-1731.

Chen HF, Chen P, Li CY (2011) Risk of malignant neoplasm of the pancreas in relation to diabetes: a population-based study in Taiwan. Diabetes Care 34(5): 1177-1179.

Chodick G, Heymann AD, Rosenmann L, Green MS, Flash S, Porath A, Kokia E, Shalev V (2010) Diabetes and risk of incident cancer: a large population-based cohort study in Israel. Cancer Causes Control 21(6): 879-887.

Cornier MA, Dabelea D, Hernandez TL, Lindstrom RC, Steig AJ, Stob NR, Van Pelt RE, Wang H, Eckel RH (2008) The metabolic syndrome. Endocr Rev 29(7): 777-822.

Coughlin SS, Calle EE, Teras LR, Petrelli J, Thun MJ (2004) Diabetes mellitus as a predictor of cancer mortality in a large cohort of US adults. Am J Epidemiol 159(12): 1160-1167.

Farrow B, Evers BM (2002) Inflammation and the development of pancreatic cancer. Surg Oncol 10(4): 153-169. 
Frye JN, Inder WJ, Dobbs BR, Frizelle FA (2000) Pancreatic cancer and diabetes: is there a relationship? A case-controlled study. Aust NZ J Surg 70: 722-724.

Gapstur SM, Gann PH, Lowe W, Liu K, Colangelo L, Dyer A (2000) Abnormal glucose metabolism and pancreatic cancer mortality. JAMA 283: 2552-2558.

Ghaneh P, Costello E, Neoptolemos JP (2007) Biology and management of pancreatic cancer. Gut 56(8): 1134-1152.

Giovannucci E, Harlan DM, Archer MC, Bergenstal RM, Gapstur SM, Habel LA, Pollak M, Regensteiner JG, Yee D (2010) Diabetes and cancer: a consensus report. CA Cancer J Clin 60(4): 207-221.

Giovannucci E, Michaud D (2007) The role of obesity and related metabolic disturbances in cancers of the colon, prostate, and pancreas. Gastroenterology 132(6): 2208-2225.

Hemminki K, Li X, Sundquist J, Sundquist K (2010) Risk of cancer following hospitalization for type 2 diabetes. Oncologist 15(6): 548-555.

Hosmer DW, Lemeshow S (2000) Applied Logistic Regression. 2nd edn. Wiley-Interscience Publication.

Inoue M, Iwasaki M, Otani T, Sasazuki S, Noda M, Tsugane S (2006) Diabetes mellitus and the risk of cancer: results from a large-scale population-based cohort study in Japan. Arch Intern Med 166(17): 1871-1877.

Inoue $\mathrm{M}$, Tajima $\mathrm{K}$, Takezaki $\mathrm{T}$, Hamajima $\mathrm{N}$, Hirose $\mathrm{K}$, Ito $\mathrm{H}$, Tominaga $\mathrm{S}$ (2003) Epidemiology of pancreatic cancer in Japan: a nested case-control study from the Hospital-based Epidemiologic Research Program at Aichi Cancer Center (HERPACC). Int J Epidemiol 32: 257-262.

Jee SH, Ohrr H, Sull JW, Yun JE, Ji M, Samet JM (2005) Fasting serum glucose level and cancer risk in Korean men and women. JAMA 293(2): 194-202.

Khan M, Mori M, Fujino Y, Shibata A, Sakauchi F, Washio M, Tamakoshi A. Japan Collaborative Cohort Study Group (2006) Site-specific cancer risk due to diabetes mellitus history: evidence from the Japan Collaborative Cohort (JACC) Study. Asian Pac J Cancer Prev 7(2): 253-259.

Lam EK, Batty GD, Huxley RR, Martiniuk AL, Barzi F, Lam TH, Lawes CM, Giles GG, Welborn T, Ueshima H, Tamakoshi A, Woo J, Kim HC, Fang X, Czernichow S, Woodward M. Asia Pacific Cohort Studies Collaboration (2011) Associations of diabetes mellitus with site-specific cancer mortality in the Asia-Pacific region. Ann Oncol 22(3): 730-738.

Larsson SC, Permert J, Håkansson N, Näslund I, Bergkvist L, Wolk A (2005) Overall obesity, abdominal adiposity, diabetes and cigarette smoking in relation to the risk of pancreatic cancer in two Swedish population-based cohorts. Br J Cancer 93(11): 1310-1315.

Lee DF, Kuo HP, Chen CT, Wei Y, Chou CK, Hung JY, Yen CJ, Hung MC (2008) IKKbeta suppression of TSC1 function links the mTOR pathway with insulin resistance. Int J Mol Med 22(5): 633-638.

Lee MS, Hsu CC, Wahlqvist ML, Tsai HN, Chang YH, Huang YC (2011) Type 2 diabetes increases and metformin reduces total, colorectal, liver and pancreatic cancer incidences in Taiwanese: a representative population prospective cohort study of 800,000 individuals. BMC Cancer 11: 20.

Lin CC, Chiang JH, Li CI, Liu CS, Lin WY, Hsieh TF, Li TC (2014) Cancer risks among patients with type 2 diabetes: a 10-year follow-up study of a nationwide population-based cohort in Taiwan. BMC Cancer 14: 381.
Lin Y, Tamakoshi A, Kawamura T, Inaba Y, Kikuchi S, Motohashi Y, Kurosawa M, Ohno Y (2002) Risk of pancreatic cancer in relation to alcohol drinking, coffee consumption and medical history: findings from the Japan collaborative cohort study for evaluation of cancer risk. Int J Cancer 99: 742-746.

Liu CY, Hung YT, Chuang YL, Chen YJ, Weng WS, Liu JS, Liang KY (2006) Incorporating development stratification of Taiwan Townships into sampling design of large scale health interview survey. J Health Manag 4: $1-22$.

Lund Nilsen TI, Johnsen R, Vatten LJ (2000) Socio-economic and lifestyle factors associated with the risk of prostate cancer. Br J Cancer 82: 1358-1363.

Matsubayashi H, Maeda A, Kanemoto H, Uesaka K, Yamazaki K, Hironaka S, Miyagi Y, Ikehara H, Ono H, Klein A, Goggins M (2011) Risk factors of familial pancreatic cancer in Japan: current smoking and recent onset of diabetes. Pancreas 40(6): 974-978.

Mickey RM, Greenland S (1989) The impact of confounder selection criteria on effect estimation. Am J Epidemiol 129(1): 125-137.

Ministry of Health and Welfare (2012) Statistical Annual Report of Medical Care: National Health Insurance, 2012. http://www.mohw.gov.tw/EN/ Ministry/Statistic.aspx?f_list_no $=474$.

Ogunleye AA, Ogston SA, Morris AD, Evans JM (2009) A cohort study of the risk of cancer associated with type 2 diabetes. $\mathrm{Br} J$ Cancer 101(7): 1199-1201.

Rulyak SJ, Lowenfels AB, Maisonneuve P, Brentnall TA (2003) Risk factors for the development of pancreatic cancer in familial pancreatic cancer kindreds. Gastroenterology 124: 1292-1299.

Silverman DT (2001) Risk factors for pancreatic cancer: a case-control study based on direct interviews. Teratogen Carcinogen Mutagen 21: 7-25.

Stevens RJ, Roddam AW, Spencer EA, Pirie KL, Reeves GK, Green J, Beral V. Million Women Study Collaborators (2009) Factors associated with incident and fatal pancreatic cancer in a cohort of middle-aged women. Int J Cancer 124(10): 2400-2405.

Stolzenberg-Solomon RZ, Pietinen P, Taylor PR, Virtamo J, Albanes D (2002) Prospective study of diet and pancreatic cancer in male smokers. Am J Epidemiol 155: 783-792.

Tseng CH, Chen CJ, Landolph Jr JR (2012) Diabetes and cancer: epidemiological, clinical, and experimental perspectives. Exp Diabetes Res 2012: 101802.

Xue F, Michels KB (2007) Diabetes, metabolic syndrome, and breast cancer: a review of the current evidence. Am J Clin Nutr 86(3): s823-s835.

Yang YX (2009) Do diabetes drugs modify the risk of pancreatic cancer? Gastroenterology 137(2): 412-415.

This work is published under the standard license to publish agreement. After 12 months the work will become freely available and the license terms will switch to a Creative Commons AttributionNonCommercial-Share Alike 3.0 Unported License. 